\title{
TN\&D per la nefrolitiasi: valutazioni critiche ad un articolo della recente letteratura scientifica
}

\author{
Introduzione
}

\section{Marco Lombardi}

S.S. Emodialis, Ospedale del Mugello e S.C. Nefrologia e Dialisi, Ospedale S.M. Annunziata, Azienda Sanitaria di Firenze, Firenze

\begin{abstract}
... "Intuisco che gli studiosi e i teorici non sono santi di cui lei è devoto" dissi. "Non sono devoto di nessun santo, amico mio, e men che meno di quelli che si canonizzano da soli o tra di loro. La teoria è la pratica degli impotenti. Le suggerisco di lasciar da parte gli enciclopedici e i loro articoli di andare direttamente alle fonti."...
\end{abstract}

Carlos Ruiz Zafon, Il gioco dell'angelo. Oscar Mondadori, Milano, 2008; pagg. 201-2.

Nel 2013 è comparsa su Annals of Internal Medicine una review sul trattamento medico della nefrolitiasi (NL) (1). Si tratta di una metanalisi che, sulla base dei risultati di $28 \mathrm{RCT}$, si propone di valutare l'efficacia della terapia medica della NL, cioè della sua capacità di prevenirne le recidive (2).

I risultati dello studio sono a dir poco sconfortanti. In pazienti che hanno avuto un singolo episodio di NL calcica esiste solo una debole evidenza per l'efficacia della terapia idropinica (RR 0.45). In pazienti con una storia di NL calcica recidivante lo studio indica invece solo una bassa evidenza per i benefici offerti dalla ridotta assunzione dei cosiddetti "soft-drinkss" acidificati con acido fosforico (RR 0.83) e una moderata evidenza per i vantaggi di una terapia con tiazidico (RR 0.52) o citrato (RR 0.25) o allopurinolo (RR 0.59) ma senza che sia possibile ottenere ulteriori vantaggi da una qualsiasi associazione di tali farmaci. Inoltre, tutti gli "scarsi" benefici dei presidi sopraddetti sono evidenti solo "contro" placebo o la semplice osservazione clinica.

Come se non bastasse, la review affossa l'utilità sia degli esami biochimici (calcio, ossalato, citrato, $\mathrm{Mg}$, fosforo e pH urinario) sia dello studio della composizione del calcolo ai fini di ottenere un trattamento più efficace per la prevenzione delle recidive.

Sulla base della mia personale esperienza con i pazienti affetti da calcolosi renale, non posso che essere in disaccordo con quanto affermato nella metanalisi. Per questo ho sentito la necessità di chiedere un parere critico, un'opinione, ad un gruppo variegato di esperti della materia, comprendente epidemiologi, nefrologi, internisti, MMG e urologi.

Indirizzo dell'Autore:

Dr. Marco Lombardi

S.C. Nefrologia \& Dialisi, Ospedale S.M. Annunziata, Firenze

e Direttore S.S. Emodialisi, Ospedale del Mugello

Via della Resistenza 60, Borgo San Lorenzo

500032 Firenze

lombardim@tin.it

\section{Bibliografia}

1. Fink HA, Wilt TJ, Eidman KE, et al. Medical management to prevention recurrent nephrolithiasis in adults: a systematic review for an American College of Physicians. Clinical guideline. Ann Intern Med 2013; 158(7); 535-43.

2. Marangella M. I compiti del nefrologo nella nefrolitiasi. G Ital Nefrol 2005; 22: 16-27. 